Nasal CPAP in OSA

\section{How best to determine optimal nasal CPAP in patients with OSAH?}

\section{A Mulgrew, J A Fleetham}

\section{Optimal CPAP pressure can be determined without in-laboratory CPAP titration. Doubt remains as to whether long term CPAP treatment reduces blood pressure in patients with OSAH}

$\mathrm{N}$ asal continuous positive airway pressure (CPAP) is the primary treatment for symptomatic patients with obstructive sleep apnoeahypopnoea (OSAH). The best method for determining the optimal CPAP pressure is an ongoing subject of scrutiny. Both the American Academy of Sleep Medicine $^{1}$ and the American Thoracic Society $^{2}$ recommend supervised CPAP titration during overnight polysomnography, based on the premise that the early establishment of optimal CPAP pressure will improve compliance with treatment. However, a recent large multicentre study ${ }^{3}$ has challenged the validity of in-laboratory CPAP titration by showing that this approach has no advantage when compared with ambulatory methods. In this issue of Thorax, West and colleagues ${ }^{4}$ further advance the case for simpler methods of CPAP pressure determination in the home.

OSAH is a common condition for which there is often limited access to appropriate diagnostic testing. ${ }^{5}$ A number of strategies have been proposed to streamline the diagnosis and treatment of patients with OSAH. Predictive algorithms help to speed diagnosis and perhaps identify patients likely to respond to CPAP. ${ }^{67}$ The widespread use of overnight home monitoring such as oximetry has improved access to diagnostic testing, and this approach is increasingly supported by published reports. $^{89}$ Several algorithm based approaches have been used with varying degrees of success to determine the optimal CPAP pressure. $^{310}$ Autotitrating CPAP machines have proved to be effective both in determining the effective pressure ${ }^{11}$ and as a treatment modality. ${ }^{12}$ Recent clinical trial data indicate that patients with a high clinical likelihood of OSAH diagnosed and treated in an entirely ambulatory setting are at no disadvantage compared with those given standard care. $^{13}$

The added expense of autotitrating CPAP machines is a burden which not all patients or healthcare providers are willing to bear. In this context, West and colleagues studied three methods of CPAP pressure determination and found that patient outcomes were comparable whether autotitration, fixed pressure following 1 week of autotitration, or fixed pressure determined by algorithm were used. No difference was noted in subjective or objective measures of sleepiness between the three patient groups during the 6 month study. Improvements in quality of life were similar with all three strategies used to determine and deliver CPAP treatment.

Compliance with CPAP is an additional important measure of patient satisfaction. All patients received CPAP education and follow up in a nurse supervised clinic. CPAP usage was excellent in the three patient groups, re-emphasising the importance of interventions to improve compliance with CPAP, ${ }^{14}$ particularly when treatment is initiated outside a sleep laboratory setting. There was no decrease in CPAP compliance over 6 months in any of the three patient groups. Although the potential cost savings of this approach the expense of an overnight titration study has been avoided, with potential additional savings related to use of more basic CPAP machines for long term treatment.

West and colleagues did not bring their patients back to the sleep laboratory to compare sleep parameters across the groups, but instead focused on clinically relevant patient centred outcomes. Mean values of the apnoeahypopnoea index as determined by the Auto-Set CPAP machine were similar in all patient groups. It should be noted that a wide range of residual sleep disordered breathing in the algorithm patient group may indicate difficulties for individual patients in whom CPAP pressure is determined by this method.

Is residual sleep disordered breathing of any significance if the patient is symptomatically improved with CPAP have not been calculated, it is clear that treatment? Why risk pressure related CPAP side effects if daytime sleepiness has been treated and quality of life is improved? Many physicians attempt to completely relieve OSAH because of a concern that persistent sleep disordered breathing may be responsible for an increased risk of cardiovascular disease. A number of cohort studies indicate an increased risk of cardiovascular events in untreated patients with OSAH, which improve with CPAP treatment. ${ }^{15}$ It is possible to trace a pathway from the inflammatory signal through several intermediate inflammatory risk factors and on to measures of atheromatous disease in patients with OSAH. Inflammatory markers known to be associated with a risk of cardiovascular disease are increased in patients with OSAH. Concurrently, there is overactivity of the sympathetic nervous system which can be shown by measuring circulating and excreted catecholamines. Vascular endothelial dysfunction and increased carotid atherosclerotic plaque formation have been demonstrated in patients with OSAH. Irrespective of whether the signal is inflammatory or physiological, the causal association between OSAH and systemic hypertension is fundamental to the potential relationship between OSAH and the premature development of cardiovascular disease. Unlike inflammatory theories which are still speculative, it is well established that OSAH is independently associated with systemic hypertension when confounding factors are taken into account.

West and colleagues studied 98 patients over 6 months and 24 hour ambulatory blood pressure was one of their outcome variables. Although neither controlled nor powered to assess changes in blood pressure with CPAP treatment, this is one of very few studies to assess the long term effects of CPAP therapy on blood pressure. After 2 months, patients using auto-CPAP and those in whom pressures were fixed after 1 week of auto-CPAP had an expected but modest decrease in blood pressure. Patients treated with an algorithm based approach showed no change in blood pressure despite improvement in all other outcome measures of daytime sleepiness and quality of life. At 6 months the mean blood pressure in all three treatment groups was the same or higher than at baseline, despite persistent improvement in all other outcome measures. The patients in this study had moderate to severe OSAH. Previous trial data indicate that, as such, they are the patients most likely to obtain a blood pressure response to CPAP treatment. It could be argued that complete relief of sleep disordered 
breathing may not have been achieved Becker et $a l^{16}$ emphasised the need for complete resolution of OSAH to reduce blood pressure in their randomised trial. West and colleagues did not confirm complete relief of OSAH with CPAP, but their treatment was sufficient to reduce blood pressure at 2 months and all other outcomes at 6 months.

A number of short term randomised controlled trials have shown a significant fall in blood pressure with CPAP treatment, but there have been no controlled studies over longer periods when a reduction in blood pressure would have greater clinical significance. Hedner et $a l^{17}$ studied cardiovascular outcomes after 12 months of CPAP treatment and found no change in blood pressure despite normalisation of catecholamine levels. In contrast, long term follow up of patients with OSAH and pre-existing hypertension has shown a reduction in blood pressure with CPAP treatment. ${ }^{18}$ Hypertensive patients had the greatest decrease in blood pressure in a short term randomised controlled trial performed by Pepperell et al. ${ }^{19}$ There is some evidence that the reduction in sympathetic activity attributable to CPAP occurs to a much more significant degree in patients with hypertension. ${ }^{20}$ Information concerning pre-existing hypertension or antihypertensive treatment was not available in the study by West and colleagues.

CPAP is clearly indicated for symptomatic patients with OSAH and the optimal pressure can be determined without in-laboratory CPAP titration. However, many relatively asymptomatic patients with OSAH are prescribed CPAP in the expectation that this wil improve their long term cardiovascular health. Barnes and co-workers looked specifically at this issue and found no beneficial blood pressure effects in patients with mild OSAH treated with
CPAP. ${ }^{21}$ The blood pressure data in the study by West and colleagues introduce further doubt as to the potential benefit of treating asymptomatic patients with OSAH. Additional randomised controlled trials are required in patients with OSAH and few daytime symptoms to determine whether CPAP improves long term cardiovascular health including blood pressure.

Thorax 2006;61:186-187. doi: 10.1136/thx.2005.050625

\section{Authors' affiliations}

A Mulgrew, J A Fleetham, Respiratory

Division, Vancouver Hospital, Vancouver, Canada V5Z 3J5

Correspondence to: Dr J A Fleetham,

Respiratory Division, Vancouver Hospital, Vancouver, Canada V5Z 3J5; john.fleetham@ vch.ca

Competing interests: none declared.

\section{REFERENCES}

1 Polysomnography Task Force, American Sleep Disorders Association Standards of Practice Committee. Practice parameters for the indications for polysomnography and related procedures. Sleep 1997;20:406-22.

2 American Thoracic Society. Indications and standards for use of nasal continuous positive airway pressure (CPAP) in sleep apnea syndromes. Official statement adopted March 1944. Am J Respir Crit Care Med 1994; 150:1738-45

3 Masa JF, Jimenez A, Duran J, et al. Alternative methods of titrating continuous positive airway pressure: a large multicenter study. Am J Respir Crit Care Med 2004;170:1218-24.

4 West SD, Jones DR, Stradling JR. Comparison of three ways to determine and deliver pressure during nasal CPAP therapy for obstructive sleep apnoea. Thorax 2006;61:226-31.

5 Flemons WW, Douglas NJ, Kuna ST, et al. Access to diagnosis and treatment of patients with suspected sleep apnea. Am J Respir Crit Care Med 2004; 169:668-72.

6 Lam B, Ip MS, Tench E, Ryan CF. Craniofacial profile in Asian and white subjects with obstructive sleep apnoea. Thorax 2005:60:504-10.

7 Flemons WW, Whitelaw WA, Brant R, et al. Likelihood ratios for a sleep apnea clinical prediction rule. Am J Respir Crit Care Med 1994; 150:1279-85

8 Chiner E, Signes-Costa J, Arriero JM, et al. Nocturnal oximetry for the diagnosis of the sleep apnoea hypopnoea syndrome: a method to reduce the number of polysomnographies? Thorax 1999;54:968-71

9 Vazquez JC, Tsai WH, Flemons WW, et al. Automated analysis of digital oximetry in the diagnosis of obstructive sleep apnoea. Thorax 2000;55:302-7.

10 Fitzpatrick MF, Alloway CE, Wakeford TM, et al. Can patients with obstructive sleep apnea titrate their own continuous positive airway pressure? Am J Respir Crit Care Med 2003;167:716-22.

11 Gagnadoux F, Rakotonanahary D, Martins de Araujo MT, et al. Long-term efficacy of fixed CPAP recommended by Autoset for OSAS. Sleep 1999;22:1095-9.

12 Ayas NT, Patel SR, Malhotra A, et al. Autotitrating versus standard continuous positive airway pressure for the treatment of obstructive sleep apnea: results of a meta-analysis. Sleep 2004;27:249-53.

13 Mulgrew A, Ayas N, Ryan CF. Ambulatory diagnosis and CPAP titration in patients with obstructive sleep apnea: can we take the pressure off the sleep lab? Eur Respir J 2005;26:116S.

14 Chervin RD, Theut S, Bassetti C, et al. Compliance with nasal CPAP can be improved by simple interventions. Sleep 1997;20:284-9.

15 Marin JM, Carrizo SJ, Vicente E, et al. Long-term cardiovascular outcomes in men with obstructive sleep apnoea-hypopnoea with or without treatment with continuous positive airway pressure: an observational study. Lancet 2005;365:1046-53.

16 Becker HF, Jerrentrup A, Ploch T, et al. Effect of nasal continuous positive airway pressure treatment on blood pressure in patients with obstructive sleep apnea. Circulation 2003; 107:68-73

17 Hedner J, Darpo B, Ejnell H, et al. Reduction in sympathetic activity after long-term CPAP treatment in sleep apnoea: cardiovascular implications. Eur Respir J 1995;8:222-9.

18 Mayer J, Becker H, Brandenburg U, et al. Blood pressure and sleep apnea: results of long-term nasal continuous positive airway pressure therapy. Cardiology 1991;79:84-92.

19 Pepperell JC, Ramdassingh-Dow S, Crosthwaite $\mathrm{N}$, et al. Ambulatory blood pressure after therapeutic and subtherapeutic nasal continuous positive airway pressure for obstructive sleep apnoea: a randomised parallel trial. Lancet 2002;359:204-10.

20 Heitmann J, Ehlenz K, Penzel T, et al. Sympathetic activity is reduced by nCPAP in hypertensive obstructive sleep apnoea patients. Eur Respir J 2004;23:255-62.

21 Barnes M, Houston D, Worsnop CJ, et al. A randomized controlled trial of continuous positive airway pressure in mild obstructive sleep apnea. Am J Respir Crit Care Med 2002; 165:773-80. 